\title{
Zur Wettbewerbsfähigkeit von brandenburgischen Klein- und Mittelunternehmen auf asiatischen Märkten
}

Beiträge und Ergebnisse des Wildauer Workshops „Internationale Wachstumsmärkte" am 8. Oktober 1997

\author{
Prof. Dr. Bernd Wonneberger, Dipl.- Psych. Hans Könecke
}

\section{Einführung}

Die Ziele und Ergebnisse des Forschungsprojektes „Markterschließung in Ost- und Süidostasien für kleine und mittlere Unternehmen KMU in betrieblicher Kooperation und Erprobung praxisnaher Methoden der Fachhochschulausbildung", welches von Oktober 1996 bis November 1997 an der Technischen Fachhochschule Wildau durchgefuihrt wurde, sind von den Autoren in anderen Beiträgen für die Wissenschaftlichen Beiträge der TFH Wildau ausfuihrlich dargestellt worden. Der hier genannte Titel des Forschungsprojektes formuliert nicht zuletzt den Anspruch, für kleine und mittlere Unternehmen Wege zur Erschließung asiatischer Märkte zu evaluieren. Von daher wollten wir die Ergebnisse unseres Forschungsprojektes den kooperierenden Unternehmen rasch zur Verfügung stellen. Hierzu veranstalteten wir am 8. Oktober 1997 gemeinsam mit dem Technologietransfer- und Weiterbildungszentrum an der Technischen Fachhochschule Wildau e.V. (TWZ), den Wildauer Workshop „Internationale Wachstumsmärkte - ihre Erschließung und Finanzierung“.

Für diesen Workshop verfaßten die Autoren zwei Beiträge, welche in gekürzter und aktualisierter Form darin den Abschnitten A und B dieses Aufsatzes dargestellt werden. Die Beiträge der ReferentInnen werden in Abschnitt $C$ dann zusammengefaßt wiedergegeben und kurz kommentiert. Für die Lektüre der Originalbeiträge verweisen wir den interessierten Leser auf den Tagungsband des Workshops, welcher über die Autoren dieses Aufsatzes oder das TWZ erhältlich ist.

\section{A. Kernprobleme der internationalen Wettbewerbsfähigkeit}

Wir wollen in diesem Abschnitt einige Kernprobleme der Wettbewerbsfähigkeit von kleinen und mittleren Unternehmen auf den Märkten in Ost- und Südostasien beschreiben, welche wir im Rahmen des Forschungsprojektes in Brandenburg und Berlin kennenlernten.

\section{A.1. Gewinnung qualifizierter Kontakte im Zielland}

Fast alle der von uns befragten Unternehmen erstellen Investitionsguiter oder technologieorientierte Dienstleistungen und beliefern ihre Stammkunden in Deutschland im Direktvertrieb.

Ein entscheidendes Problem bei der Markterschließung in Asien für Investitionsgüter und Dienstleistungen besteht zunächst einmal darin, daß dort andere technische Normen gelten. Einige der angebotenen Lösungen in der Meß- oder Medizintechnik sind bis dato in Asien unbekannt, so daß dort der Markt hierfür überhaupt erst geschaffen werden müßte. Letztlich benötigen die Anbieter von Investitionsgütern und technologieorientierten Dienstleistungen für ihre vielfach erklärungsbedürftigen Produkte Servicepartner im jeweiligen Zielland. Die Gewinnung qualifizierter Kontakte wurde in unserer schriftlichen Befragung als eines der größten Probleme genannt (vgl. WONNEBERGER et al. 1997a). Bei den von uns im ausführlichen, persönlichen $\mathrm{Ge}$ spräch interviewten 31 Unternehmen entstanden die internationalen Erstkontakte fast alle iiber Fachmessen der jeweiligen Branchen, Anfragen von ausländischen Importeuren, Ingenieurbüros und, last but not least, Veranstaltungen der Wirtschaftsförderung, der Fachverbände und der Außenhandelskammern. In einem (mehr oder weniger) aufwendigen Prozeß muß die Qualität verschiedener Kontakte, d. h. die Leistungsfähigkeit und das Know-How der ausländischen Partner, durch das deutsche Unternehmen geprüft werden. Für die Schulung und Betreuung von Kunden, den Verkauf und den Service komplexer Produkte sind im Auslandsmarkt Partner von hoher technischer Kompetenz erforderlich. Nach den Ergebnissen unserer Recherchen, welche durch Interviews mit größeren Stichproben von KMUs noch empirisch zu verifizieren wären, entstand bei den Autoren der Eindruck, daß die Mehrzahl der Unternehmen im internationalen Marketing anfangs auf Anfragen und Anregungen von außen (beispielsweise Anfragen von Messebesuchern, Unternehmerreisen der Brandenburgischen Außenhandelsagentur u. a. mehr) nur reagieren.

Obwohl die meisten der befragten Unternehmen seit 1990 in gewissem Sinne neu „gegründet" wurden, war nur in wenigen Fällen ein aktives internationales Marketing von Beginn an Bestandteil der Unternehmensstrategie. Zwei Mitarbeiter jener Unternehmen in Brandenburg, die ein konsequentes internationales Marketing betrieben haben, konnten wir als Referenten für unseren Workshop gewinnen (vgl. unten, Abschnitt C.1. und C.2.). Nach den Aussagen dieser Unternehmen muß die Qualität eines aktiven internationalen Marketing im Unternehmen vor Ort in Brandenburg definiert und realisiert werden, wobei für die Qualität nach unserer Auffassung im wesentlichen zwei Parameter gelten:

1. Gutes Briefing der Außenhandelskammern, Consulter, Forschungsagenturen und anderer Rechercheure, die qualifizierte Kontakte für das Unternehmen auf ausländischen Märkten finden sollen. Briefing meint die detaillierte Information der genannten Personen/Institutionen darüber, nach welchen Kriterien qualifizier- 
te Partner - Endkunden, Absatzmittler, Servicepartner - für das Unternehmen ausgewählt werden sollen.

2. Fremdsprachliche Firmen- und Produktinformationen, welche im Rahmen der Recherche auf den Zielmärkten an potentielle Partner zur Erstinformation übergeben werden können.

Aktives internationales Marketing ist nach unserem Verständnis gekennzeichnet durch die Fähigkeit, die Endkunden, Absatzmittler und ggf. Servicepartner auf verschiedenen Ländermärkten a) zu erkennen und b) gezielt selbst anzusprechen, nicht abwartend nur auf Anfragen zu reagieren. Mit welchen Maßnahmen die Fähigkeit der KMU zum aktiven internationalen Marketing verbessert werden könnte, diskutieren wir in den Abschnitten B. und C. dieses Aufsatzes.

\section{A.2. Kosten der Markterschließung in Ostasien}

\section{A.2.1 Markterschließung vor Ort - Fallbeispiel Shanghai} In der schriftlichen Befragung (vgl. WONNEBERGER et al. 1997a) hatten wir danach gefragt, welche Anschubfinanzierung über ein Jahr die Unternehmen für die Erschließung asiatischer Märkte bräuchten. Die Anworten jener Unternehmen, die bereits in Asien tätig sind (arithmetisches Mittel 120 TDM) unterschieden sich deutlich von denen jener Unternehmen, welche noch nicht in Asien tätig sind (arithmetisches Mittel 500 TDM). Da jedoch die Spannweite der Antworten in beiden Gruppen groß war, entschlossen wir uns, für die Interviews ein detaillierteres Fallbeispiel zu konstruieren.

Wir nahmen an, daß zur Vorbereitung einer längerfristigen, technischen Kooperation ein Mitarbeiter des Unternehmens für drei Monate nach Shanghai geschickt werden sollte. Die Interviewpartner sollten die Höhe verschiedener Kosten (Flug, Hotel, Telekommunikation u. a. m.) dieses Auslandsaufhaltes schätzen. Kosten, die in Brandenburg anfallen (Fortzahlung des Mitarbeitergehaltes u. a.), blieben außerhalb der Betrachtung.

Die Unternehmen schätzten (mit geringer Spannweite) die Kosten eines dreimonatigen Aufenthaltes in Shanghai in der Summe auf 30 TDM. Dies entspräche dem oben genannten Jahreswert von 120 TDM; diese Kosten entstïnden bei der Buchung von Deutschland aus.

\section{A.2.2 Gebühren und Finanzierungskosten}

Nach dem Wissen der Autoren betragen die Gebühren für die Eröffnung und Abwicklung eines Dokumentenakkreditivs bei deutschen Geschäftsbanken mindestens 200 DM. Hinzu kommen die IHK-Gebühren für die Ursprungszeugnisse, sowie Gebühren für die Ausfertigung der Zollpapiere (durch den Spediteur). Summa summarum entstehen hier, unabhängig vom tatsächlichen Auftragswert, bereits fixe Kosten von $500 \mathrm{DM} u$. m. (vgl. HENNING/SCHIEWE 1997). Ferner sind die gewichtsund volumenabhängigen Transportkosten sowie das Delkredere bis zum Eingang der Kundenzahlungen zu finanzieren. All dies verteuert den Preis deutscher Waren auf dem Weltmarkt, so daß unter den geschilderten Bedingungen kleinere Auslandaufträge (mit einem Wert unter 100 TDM) weder für die Unternehmen als direkte Lieferung vom Hersteller zum ausländischen Kunden, noch für die Banken attraktiv sind. Die Transport- und Finanzierungsrisiken kleinerer Aufträge sollten eher übernommen von speziell erfahrenen Handelshäusern und -kommissionären im Außenhandel übernommen werden (vgl. WONNEBERGER/SWAT et al. 1998).

\section{A.2.3 Kalkulation von Angebotspreisen}

(in ausländischer Währung)

Wir sagten bereits in Abschnitt A.1., daß die befragten Unternehmen internationale Kontakte nicht von Anfang an systematisch erarbeiten. Auf den oben genannten Wegen, sogar durch Einträge in deutschen Fachadreßbüchern erreichen die kleinen und mittleren Unternehmen Brandenburgs vereinzelte Anfragen selbst aus Australien, Hongkong, Indien oder Taiwan. Zur vollen Kostendeckung müßten eigentlich alle in A.2.1. und A.2.2. genannten Positionen in den Angebotspreis eingerechnet werden, die jedoch selten in ihrem vollen Umfang anfangs von den KMU erfaßt werden. Zunächst werden nicht kostendeckende Preise angeboten - später möglicherweise übereilte Nachforderungen erhoben, die nur selten bei den ausländischen Kunden durchzusetzen sind. Wie schwierig zudem eine Absicherung des Währungsrisikos ist, zeigt der tägliche Blick in die Wirtschaftspressse.

\section{A.3. Interkulturelle Vorurteile gegenüber Ost und Südostasien, insbesondere der VR China}

Die meisten der von uns im Sommer 1997 befragten Unternehmen hatten sich zumindest über einzelne asiatische Märkte grob informiert, die Suche nach Detailinformationen und weiteren Kontakten in Asien dann jedoch abgebrochen. Gelegentliche Kontakte nach Asien, die durch Dritte vermittelt worden waren, konnten oder wollten die Unternehmer nicht weiter verfolgen - zumeist mit der Begründung, daß das finanzielle und zeitliche Budget zur Intensivierung dieser Kontakte momentan nicht vorhanden sei. In der Interviewsituation wurden nur vier Einzelmeinungen geäußert, welche der Interviewer als Vorurteile klassifizieren würde. Ein Unternehmer sagte: „Geschäftspartner in Europa oder Nordamerika stehen mir mental näher, ihr Verhalten ist für mich durchsichtiger". Ein weiterer Unternehmer war der Ansicht, daß „die Chinesen sowieso Weltmeister im Ideenklau" seien, die ohne Rücksicht auf Urheberrechte jede Innovation nachkupfern würden. Ein dritter Unternehmer sagte, daß „die Entscheidungswege in der chinesischen Bürokratie zu langwierig “ wären. Wiederum ein vierter Unternehmer sagte, daß die geographische Distanz und der Zeitunterschied zu groß wären. „In China komme ich nach 20 Stunden Flugzeit nicht gerade topfit an. Wenn ich dagegen Geschäfte in Polen mache, bin ich innerhalb von 4 Stunden vor Ort und bringe die auf Trab, falls es bei meinen polnischen Partnern mal wieder klemmt".

Die genaue Wertigkeit derartiger Vorurteile für die Länderwahl der Unternehmen in Berlin und Brandenburg wäre durch weitere Untersuchungen (mit entsprechendem Focus und Design) noch zu evaluieren. Kulturelle Präferenzen deutscher Manager für Europa und Nordamerika sind ja bereits in den Untersuchungen von 
DICHTL, HOLZMÜLLER, KÖGLMAYR, MÜLLER u. a. festgestellt worden, allerdings in einem ganz anderen ökonomischen und weltpolitischen Umfeld. In Brandenburg und Berlin sind viele Unternehmer unter den Bedingungen des Sozialismus groß geworden, so daß mit einem verfeinerten Instrumentarium noch zu erforschen wäre, wieweit wir hier, trotz anderer Biographien und ökonomisch-politischer Bedingungen, die gleichen interkulturellen Präferenzen und Vorurteile finden, wie bei anderen Untersuchungen im deutschen Sprachraum.

\section{A.4. Kenntnisse von Fremdsprachen}

Für eine Prüfung und ggf. Intensivierung ausländischer Geschäftskontakte haben die befragten Unternehmen nach unserer Auffassung zu wenig oder gar kein Personal. Wenn überhaupt, dann haben nur die Geschäftsführer und die Entwicklungsingenieure Kenntnisse der englischen Fachsprache der jeweiligen Technik. In den regelmäßig erscheinenden Geschäftskontakten der Bundesstelle für Außenhandelsinformationen wird in 80 Prozent der Fälle Englisch als Korrespondenzsprache angegeben. Allein das Zeitbudget des Managements von KMU ist für eine kontinuierliche Korrespondenz in englischer Sprache zu gering. Für die permanente Betreuung ausländischer Servicepartner und Kunden wäre es nach unserer Auffassung notwendig, daß auch die Mitarbeiter beispielsweise in der Telefonzentrale oder im Sekretariat des Unternehmens zumindest soweit die englische Sprache beherrschen, daß sie die Wünsche der ausländischen Anrufer erkennen und das Gespräch an kompetente Mitarbeiter des eigenen Unternehmens weiterleiten können.

\section{A.5. Internationale Marketing- und Management- kompetenz in KMU}

KMU bevorzugen (wegen ihrer geringen finanziellen Ressourcen) im internationalen Geschäft die Zusammenarbeit mit Handelshäusern im Zielland (ausführlicher dazu WONNEBERGER/SWAT et al. 1998). Dadurch erwerben die Unternehmen jedoch selbst zu wenig Kompetenzen im internationalen Geschäft - Marktchancen auf Auslandsmärkten werden nicht systematisch erkundet, ebensowenig sind die Risiken einzelner Ländermärkte im Detail bekannt. Neben der bereits in A.4. genannten Fremdsprachenkompetenz sind für das internationale Geschäft Kenntnisse der verschiedenen Rechtssysteme, Absatzwege, Konsumentengewohnheiten, Logistik, Währungsentwicklungen, Zollabwicklung u. a. m. wesentlich. Das Wissen hierüber, die Kompetenzen hierin sind nur begrenzt durch Lehrbüicher, Seminare oder die Publikationen der Bundesstelle für Außenhandelsinformationen vermittelbar, hingewiesen sei an dieser Stelle nur einmal auf die sich immer wieder ändernden Zollbestimmungen sowohl der EU wie vieler anderer Länder. Von daher sind selbst in kleinen Unternehmen mit schmalen, tiefen Sortimenten eigene Mitarbeiter für das internationale Geschäft von dem Moment an erforderlich, in dem mehrere Ländermärkte in verschiedenen Wirtschaftszonen außerhalb der EU bearbeitet werden. Als Investition in die Zukunft werden Mitarbeiter für das Auslandsgeschäft in KMU vorab nur selten eingestellt.
Zunächst bearbeitet nur der Geschäftsführer oder jener Mitarbeiter die Auslandsmärkte, der bis dato auch den Inlandsmarkt bearbeitete. Wir konnten bei unseren Besuchen in den brandenburgischen Unternehmen mehrfach beobachten, daß eine größere Zahl von Auslandsanfragen das Zeitbudget des Geschäftsführers oder seiner Mitarbeiter überschritt.

\section{A.6. Firmeninformationen in Fremdsprachen}

Von den 31 interviewten KMU verfiigten 17 über Firmeninformationen in Englisch. Im Laufe der vergangenen Jahre ließ darüber hinaus ein Unternehmen Materialien in Arabisch, Französisch und Russisch erstellen. Von der Gründung im Jahre 1992 an erstellte nur die BE Maschinenmesser GmbH \& Co. KG Firmen- und Produktinformation in vier Fremdsprachen - Englisch, Französisch, Italienisch und Spanisch - ein Muster dieser viersprachigen Kurzinformation ist in den Tagungsmaterialien des Workshops dokumentiert. Vier Jahre nach seiner Gründung erzielte dieses Unternehmen im Jahre 1996 bereits ca. 1,5 Mio. DM Exportumsatz.

In den anderen KMU wurden bzw. werden fremdsprachliche Prospekte, Produktinformationen u. a. Materialien in der Regel erst erstellt, wenn konkrete Anfragen aus dem Ausland vorliegen. Wertvolle Zeit geht verloren, wenn dann erst noch Informationen in Fremdsprachen übersetzt und gedruckt werden müssen. Für das Fachpublikum reicht zumeist die Information und Kommunikation in Englisch aus (vgl. oben A.4.). Sofern ein breiteres Publikum angesprochen werden soll, halten die Autoren die Bereitstellung von Informationen in weiteren Weltsprachen für wichtig. Nach ihrer weltweiten Verbreitung wären dies insbesondere Arabisch, Chinesisch, Französisch, Russisch und Spanisch.

\section{A.7. Nutzung von Datenbanken und Datennetzen}

Informationen über neue Ergebnisse der Forschung an Fachhochschulen und Universitäten lassen sich heutzutage rasch und effizient aus weltweit zugänglichen Datenbanken recherchieren und für das eigene Unternehmen nutzen. Das Internet bietet völlig neue Möglichkeiten, Interessenten und Kunden weltweit durch die eigenen Webseiten zu informieren oder Angebote und Produktinformationen schnell per e-mail zu versenden. 20 der 31 befragten Unternehmen haben einen Internetanschluß, der jedoch aus zwei Gründen in den kleinen und mittleren Unternehmen nur gelegentlich genutzt wird:

1. Opportunitätskosten zur Schulung der Mitarbeiter in der Netzwerktechnik,

2. im internationalen Vergleich hohe deutsche Kosten der Telekommunikation.

\section{A.8. Beteiligung an internationalen Ausschreibungen}

Größere Infrastrukturvorhaben (Energie, Verkehr, Kommunikation u. a. m.) werden durch die EU, die Weltbank und andere Institutionen im Datennetz ausgeschrieben, auch dies wiederum vorwiegend in englischer Sprache. 
Allein die EU stellt an manchen Tagen 500 Ausschreibungen (engl. „tender“) ins Netz. Kleineren deutschen Unternehmen, u. a. fachlich hoch spezialiserten Ingenieurbuiros, fehlt es auch hier wiederum an fremdsprachlich und datentechnisch qualifiziertem Personal, um a) diese Fïlle an Ausschreibungen permanent zu sichten und ggf. b) ein differenziertes Angebot abgeben zu können.

\section{B. Lösungsansätze zur Verbesserung der internationalen Wettbewerbsfähigkeit}

Nach Auffassung der Autoren sollten hierbei vor allem jene Maßnahmen zur Verbesserung der internationalen Wettbewerbsfähigkeit Priorität genießen, welche in den Unternehmen ansetzen. Die Erschließung und Gewinnung ausländischer Märkte durch kleine und mittlere Unternehmen sehen wir, wie auch andere Autoren, als „endogenen Lernprozeß“ (vgl. WONNEBERGER/SWAT et al. 1998), der abhängig ist von der Unternehmensgröße (Mitarbeiterzahl, Jahresumsatz), dem finanziellen, technologischen und Marketingpotential eines Unternehmens. In einer sich stetig weiter vernetzenden Weltwirtschaft würde es jedoch die Wettbewerbsfähigkeit auch junger, innovativer Unternehmen schmälern, wenn man nur den Zeitpunkt abwarten würde, bis die Unternehmen aus eigener Kraft den notwendigen „Reifegrad“, eine ausreichende Größe für das internationale Geschäft, erreichten.

Erfolgreiche Unternehmen versuchen heutzutage so früh wie möglich, von der Gründung an, Auslandsmärkte zu gewinnen. „Wie wurden die ,Hidden Champions‘ Weltmarktführer? Bestimmt nicht dadurch, daß sie zu Hause blieben und auf Kundenanrufe warteten. Sie gingen vielmehr in die Welt hinaus und machten ihre Produkte und ihren Service ihren Kunden verfuigbar, wo immer diese auch waren" (SIMON, 65). Genau diese Einstellung ist es, die wir aktives internationales Marketing genannt haben (vgl. oben A.1.). Immerhin konnten wir bei unseren Interviews in den brandenburgischen Unternehmen feststellen, daß 8 von 31 befragten Unternehmen in ihrer Selbsteinschätzung sagten, sie seien im nationalen, ja sogar im internationalen Wettbewerb derzeit technologisch führend. Auch diese Unternehmen können - vor allem wegen geringer personeller Kapazitäten - im Augenblick gar nicht alle Marktchancen nutzen (vgl. oben A.4. und A.5.). Die meisten der von uns befragten Unternehmen haben zu wenige Mitarbeiter mit den für das internationale Geschäft wichtigen Kenntnissen von Fremdsprachen und Datentechnik. Letztlich entwickelten wir in unseren Gesprächen mit den Unternehmen Ideen, um den personellen Engpaß in den Unternehmen durch die folgenden Ansätze zu lösen.

\section{B.1. Innovations- und Marketingassistenten-Modell versuch „tenders \& projects management by contract"}

Die in den Abschnitten A.4. bis A.8. beschriebenen Know-How-Defizite könnten durch den Einsatz von (daten-)technisch, betriebswirtschaftlich und fremdsprachlich qualifizierten Innovations- und Marketing- assistenten entscheidend verringert werden. Diese Assistenten sollten in einem (von der Gründung an international orientierten) Unternehmen folgende Aufgaben übernehmen:

1. Informationen über technische Entwicklungen zur Sicherung der internationalen Wettbewerbsfähigkeit,

2. Marktinformationen zu den wichtigsten Ländermärkten,

3. Sichtung internationaler Ausschreibungen (vgl. A.8.),

4. Beantwortung weltweiter Kundenanfragen, Erstellung von Angeboten u. a. bei internationalen Ausschreibungen.

Nach Auffassung einiger der von uns befragten Unternehmen sollten diese „IuM-Assistenten“ auf einer soliden technischen Basis qualifiziert werden, da die befragten Unternehmen (Anbieter von Investitionsgütern und/ oder gewerblichen Dienstleistungen) ausschließlich Auftragsfertigung betreiben -die Kundengewinnung und Auftragsbearbeitung erfordert hierbei ein gutes technisches Wissen. Rein betriebswirtschaftlich und fremdsprachlich qualifizierte Mitarbeiter sind in erster Linie einsetzbar in Unternehmen, welche standardisierte, normierte Produkte (quasi aus dem Katalog heraus) verkaufen.

Ein mittelständiges Unternehmen der Elektroindustrie sah den entscheidenden Engpaß im internationalen Geschäft darin, internationale Ausschreibungen überhaupt auswerten und ggf. Angebote abgeben zu können. Nur einer von 220 Mitarbeitern sei dazu in der Lage - sofern er nicht gerade andere Aufgaben im Unternehmen zu erledigen hätte. Für eine Überwindung dieses Engpasses schlug der Unternehmer die zeitlich befristete Beschäftigung von Fachpersonal vor, welches internationale tender (Ausschreibungen) auswählt und hierfür Angebote erstellt. Als Träger schlug der Unternehmer eine „neutrale Agentur“ (beispielsweise eine Fachhochschule) vor, welche den Unternehmen dieses Personal zunächst kostenlos zur Verfügung stellt. Im Falle des Erfolges, also bei der Gewinnung eines Auftrages, hätte dann das Unternehmen ein Erfolgshonorar an die Agentur zu zahlen.

Nach Auffassung der Autoren könnte diese Idee tatsächlich geeignet sein, die internationale Vermarktung technisch komplexer Investitionsgüter und Dienstleistungen aus der Region Berlin-Brandenburg zu intensivieren, da diese Produkte nicht so einfach wie Konsumgüter, Rohstoffe, Werkstoffe und Zubehörkomponenten über internationale Handelshäuser vermarktet werden können. Wir regen deshalb an, diese Idee aus Unternehmerkreisen in einem Modellversuch „tenders \& projects management by contract" weiter zu verfolgen. In Gesprächen mit den Fachverbänden der gewerblichen Wirtschaft, dem Landesarbeitsamt Berlin-Brandenburg, der Wirtschaftsförderung, dem World Trade Center und weiteren Institutionen wären insbesondere zu bestimmen

a) der tatsächliche Personalbedarf in den Unternehmen der Region und

b) die organisatorische und rechtliche Trägerschaft eines solchen Modellversuches. 


\section{B.2. Regionale Zulieferbörsen}

Der Schritt auf den Weltmarkt fällt insbesondere jungen Unternehmen wesentlich leichter, wenn sie über eine solide Basis im Heimatland verfügen. Bei unseren Interviews ist uns aufgefallen, daß etliche Unternehmen in Brandenburg nur in geringem Umfang konkret wissen, wer hier als Lieferant oder Dienstleister für sie vor Ort in Frage kommt. Um die Wirtschaftsbeziehungen in dieser Region zu intensivieren, sollte die Veranstaltung von Zulieferbörsen, die Bildung von Bietergemeinschaften für Infrastrukturprojekte und andere Formen der regionalen Wirtschaftskooperation noch intensiver gefördert werden. Ein gutes Beispiel bieten hier die Gründung der Arbeitsgemeinschaft Schienenverkehrstechnik Brandenburg im August 1997 oder die Anbietergemeinschaft Kraftwerkstechnik des World Trade Center Frankfurt (Oder).

\section{B.3. Schaffung von Referenzprodukten und -projekten für neue Technologien - Internationale Vermark- tung von Beginn der Markteinführung an}

Die Notwendigkeit einer Verwaltungsreform sowie die Verringerung der unüberschaubaren Vielzahl rechtlicher Vorschriften, die in Deutschland bei betrieblichen Aktivitäten zu beachten sind, ist zwar oft genug politisch gefordert worden; bei den Behörden in Bund, Ländern und Gemeinden ist bisher jedoch im Detail zu wenig realisiert worden. Um so dringlicher wird die Abschaffung derartiger Vorschriften, wenn diese den Einsatz energie- und ressourcenschonender Technologien behindern. Die Schaffung von Referenzprodukten und -projekten für neue Technologien sollte nicht erst in den Dimensionen eines Transrapid, sondern genauso für kleine, intelligente Entwicklungen mit dem Ziel einer nachhaltigen Wirtschaft möglich sein. Für deren frühestmögliche internationale Vermarktung benötigen wir ferner eine weitestgehende Verzahnung von Technologie- und Außenwirtschaftsförderung auf Bundes- wie auf Landesebene.

\section{B.4. Internationale Kontakte der Fachhochschulen und Universitäten}

Detaillierte Recherchen auf ausländischen Märkten, die wesentlich tiefer gehen als die Publikationen der Bundesstelle für Außenhandelsinformationen, erfordern die Entsendung eigenen Personals, anderenfalls die Beauftragung eines international tätigen Consultants oder Researchers. Beide Varianten erfordern finanzielle Vorleistungen des Unternehmens in beträchtlicher Höhe.

Informationen über Auslandsmärkte ließen sich auch (gelegentlich sogar kostengünstiger) durch die internationalen Kontakte der Studenten, Professoren und anderer Mitarbeiter an deutschen Hochschulen und Fachhochschulen gewinnen. Durch Fachkongresse, Studentenaustausch und andere internationale Aktivitäten entsteht hier tagtäglich ein Netzwerk, in welchem Ergebnisse aus Forschung und Wissenschaft verschiedenster Disziplinen ausgetauscht werden. Darüber hinaus wird der Einblick in andere Kulturen, Mythen, Religio- nen, Sitten und Gebräuche möglich, welche für die Markterschließung wichtig sein können (vgl. HOFSTEDE 1993, USUNIER + WALLISER 1993). Möglicherweise entstehen auch hierüber potentiell qualifizierte Kontakte zu Entscheidungsträgern in den Regierungen, Fachverbänden und Unternehmen der Zielmärkte.

In diesem Sinne sollten, trotz aller Kürzungen der Etats, unsere Professoren und Studierenden weiterhin die Chance erhalten, weltweit tragfähige Kontakte und Beziehungen auf ihrem jeweiligen Fachgebiet zu entwikkeln und zu pflegen.

\section{B.5. Weltweit anerkannte Studiengänge der deut- schen Fachhochschulen und Universitäten}

„Im Vergleich zu den Vereinigten Staaten, Australien und Großbritannien haben deutsche Universitäten nach Angaben des Bildungsministeriums an internationaler Attraktivität verloren. Dies gilt insbesondere für Studenten aus Asien und Amerika. Unter den zwei Millionen Studenten an deutschen Hochschulen sind 140.000 Ausländer, also sieben Prozent. An Eliteuniversitäten in Amerika und England liegt der Anteil der Ausländer an der Gesamtzahl der Studenten wesentlich höher als in Deutschland. In Oxford etwa kommen 22 Prozent der Studenten von außerhalb der Europäischen Union ... Durch die Hochschulreform sollen deutsche Universitäten nun stärker international ausgerichtet werden: verkürzte Studienzeiten, weltweit gültige Abschlüsse als Bachelor und Master sowie mehrsprachige Studiengänge sind geplant" (Bztg, 1.9.1997).

Ferner hat der DAAD einen Wettbewerb der deutschen Fachhochschulen und Universitäten zum Auslandsmarketing ausgeschrieben. All diese Bemuihungen zielen letztlich in Richtung einer (Fach)hochschulausbildung in Deutschland, welche international kompetente Absolventen fur deutsche und andere Unternehmen ausbildet.

Die Ausbildung ausländischer Studenten in Deutschland kann für deutsche Unternehmen tatsächlich von strategischem Interesse sein (vgl. WONNEBERGER/SWAT et al. 1998), wenn diese Studenten später in ihr Heimatland zurückkehren und dort den Markt für ein deutsches Unternehmen erschließen. Im Laufe der Jahre werden diese Absolventen deutscher (Fach)hochschulen eventuell auch zu Entscheidungsträgern in ihren Ländern, wobei sie hoffentlich eine gewisse, positive Affinität gegenüber jenem Land, in welchem sie studieren konnten, bewahren.

\section{B.6. Exportakademie der neuen Bundesländer}

Die Autoren halten es für schwierig, bei einigen Themen sogar für unmöglich, internationale Marketing- und Managementkompetenz als reines „Bücherwissen“ zu vermitteln. Veranstaltungen der Fachverbände und anderer Institutionen zu Zoll-, Branchen-, Kultur- und Länderinformationen sind wichtig, um das notwendige Wissen a) kompakt und anschaulich zu vermitteln und b) den Erfahrungsaustausch der Unternehmen untereinander zu initiieren und zu verstärken. Nach Aussagen der durch die Autoren befragten Veranstalter auf dem Gebiet der Außenwirtschaft werden in der Region Berlin-Brandenburg 
vor allem Veranstaltungen zu Mittel- und Osteuropa nachgefragt. Veranstaltungen zu anderen Ländermärkten oder Wirtschaftsregionen (z. B. NAFTA, ASEAN) finden allein im Raum Berlin-Brandenburg oftmals nicht genügend Teilnehmer. Von daher würden sich die Autoren wünschen, daß Veranstaltungen zu den Wachstumsmärkten in Amerika oder Asien von Thüringen bis Mecklenburg-Vorpommern angekündigt würden, um so die Chancen ihrer Realisierung zu erhöhen. Bei der vergleichsweise geringen industriellen Basis in den neuen Bundesländern sollten die Programme und Veranstaltungen zur Außenwirtschaft der einzelnen Fachverbände aufeinander abgestimmt und länderübergreifend angeboten werden. Vielleicht könnte ein Teil dieser Veranstaltungen auch durch eine gemeinsame Exportagentur der neuen Bundesländer organisiert werden, welche Klaus von Dohnanyi im Sommer 1997 vorgeschlagen hat (vgl. „Lausitzer Wirtschaft“ Heft 7+8/97; „Berliner Zeitung“, 1.9.1997).

In Baden-Württemberg arbeitet die Exportakademie in Reutlingen erfolgreich mit der mittlerweile 28. Seminarreihe, welche den internationalen Erfolg der Unternehmen aus Baden-Württemberg verschiedentlich vorbereiten und fördern konnte. Für einen vergleichbaren internationalen Erfolg sind, nicht nur in Berlin und Brandenburg, insgesamt noch erhebliche Anstrengungen in den neuen Bundesländern notwendig.

\section{Beiträge und Ergebnisse des Wildauer Workshops am 8.10.1997}

Die Originalmanuskripte dieser Beiträge sind in den Tagungsmaterialien des Wildauer Workshops „Internationale Wachstumsmärkte -Ihre Erschließung und Finanzierung" dokumentiert. In diesem Aufsatz geben wir die (aus Sicht der Autoren) wesentlichen Kernaussagen der Referenten wieder.

\section{C.1. Erfolgreiche Markterschließung in Asien} Kerstin Thomsen, Exportleiterin der BE Maschinenmesser GmbH \& Co. KG, Spreenhagen

In ihrem Referat gab Frau Thomsen einen Überblick über die Geschichte ihres Unternehmens und die Entwicklung seines Sortimentes seit 1992. Die Maschinenmesser werden mit modernster Konstruktion und Fertigung a) für Maschinen- und Anlagenbauer der Lebensmitteltechnik (Hersteller), vor allem der Fleischverarbeitung, und b) für Großhändler von Maschinen und Komponenten des in a genannten Wirtschaftszweiges maßgefertigt.

Von der Gründung an bemühte sich das Unternehmen innerhalb und außerhalb Europas um die Gewinnung der genannten Kunden. Als einer der weltweit wichtigsten wurde der japanische Markt eingeschätzt, über den seit Ende 1994 zielgerichtet Informationen gesammelt wurden aus folgenden Quellen:

a) Bundesstelle für Außenhandelsinformation BfAI

b) IHK und AHK in Tokio

c) Kataloge von internationalen Fachmessen des Wirtschaftszweiges Lebensmitteltechnik

d) JETRO Berlin
Ergänzend zu ihrem Manuskript beschrieb Frau Thomsen lebendig und anschaulich die Vorbereitungen ihres Unternehmens auf den Besuch der International Food Machinery Exhibition vom 30.5. bis 3.6.1996 in Tokio. Der Messebesuch und die anschließenden Besuche bei potentiellen Kunden bzw. Vertriebspartnern in Japan wurden zielgerichtet über zwölf Monate hin vorbereitet. Hilfestellung durch Informationen über die japanische Kultur gab dabei u. a. der Asien-Referent der Wirtschaftsförderung Brandenburg GmbH.

Von Spreenhagen aus wurden vor der Reise nach Japan die Gesprächspartner über die BE Maschinenmesser informiert. Ein Teil der Korrespondenz und der Telefonate konnte in Englisch geführt werden; Visitenkarten, Katalogblätter und andere Materialien wurden in die japanische Sprache übersetzt. Den Wert des kulturellen Wissens und der Materialien in japanischer Sprache schätzt Frau Thomsen wie folgt ein: „Natürlich hängt der Erfolg eines Markteinstiegs nicht von der korrekten Form allein ab, aber eine gewisse Sicherheit und Beratung auf diesem Gebiet hilft sehr, daß man sich dann im direkten Kontakt auf das Wesentliche - nämlich das Produkt - beziehen kann" (Tagungsbeitrag, S. 5).

Parallel zu den Aktivitäten in Japan akquirierte das Unternehmen Kunden und Vertriebspartner in den USA und Lateinamerika. All dies erfordert auch heute noch erhebliche finanzielle Investitionen für die Markterschliessung, die das Eigenkapital und den Kreditrahmen eines kleinen Unternehmens stark belasten. Am Ende ihres Beitrages machte Frau Thomsen deutlich, welche Existenzhilfe das Vermarktungshilfeprogramm der Bundesregierung für ihr Unternehmen war und ist, wobei für Frau Thomsen der Erfolg resp. Mißerfolg bei der Teilnahme am Vermarktungshilfeprogramm a) von der Erfahrung und den spezifischen Länder- und Marktkenntnissen des jeweils beauftragten Projektleiters und b) von der detaillierten Information („Briefing“) des Projektleiters über die Leistungen des Unternehmens abhängig ist.

\section{C.2. Erfahrungen bei der Vorbereitung eines Joint Ventures in China}

Dr.-Ing. Peter Schulz, Prokurist der WBG Weichenwerk Brandenburg GmbH

Das Weichenwerk Brandenburg GmbH fertigt hochwertige Komponenten - Weichen - für Schienenverkehrswege. Zusammen mit seinem Hauptgesellschafter, einem Weichenwerk in Hessen, ist das Unternehmen Marktführer in Deutschland und Hauptlieferant der Deutschen Bahn AG. Deren Erstinvestitionen und Ersatzbeschaffungen an Weichen gehen seit 1990 zurück. Von daher bestand für das Weichenwerk Brandenburg $\mathrm{GmbH}$ „von Anfang“ an (seit der Privatisierung im Jahre 1990) die Notwendigkeit, Auslandsmärkte zu erschließen. Bei den (zumeist noch staatlichen) Eisenbahngesellschaften anderer Nationen stehen ausländischen Anbietern jedoch nicht von vornherein „die Türen offen“. Neben vielen anderen Aktivitäten der Markterschließung nahm der Geschäftsführer der WBG 1994 an einer (staatlich geförderten) Unternehmerrreise der Brandenburgischen Außenhandelsagentur in die VR China teil. 
Bereits bei diesem Besuch zeigte das chinesische Eisenbahnministerium starkes Interesse für die Leistungen des Unternehmens und äußerte den Vorschlag, in einem Joint Venture ein neues Weichenwerk in der Nähe von Shanghai zu errichten. Zunächst schickte die WBG ein Musterprodukt zur Erprobung nach China. Im tagtäglichen 24-Stunden-Einsatz über mehr als zwei Jahre erreichte die Weiche die dreifache Liegedauer gegenüber chinesischen Produkten.

Anläßlich des Besuches des brandenburgischen Ministerpräsidenten Stolpe wurde im Februar 1997 der Letter of Intent zur Errichtung des Joint Venture mit der Eisenbahndirektion Shanghai unterzeichnet. Nach Klärung weiterer technischer und finanzieller Fragen soll das Joint Venture Anfang 1998 in Kraft treten. Die technische Führung des Joint Ventures wird bei der WBG liegen, was auch bedeutet, daß in den nächsten Jahren wesentliche Komponenten für die chinesische Produktion aus Deutschland geliefert werden. Eine Rücklieferung der chinesischen Erzeugnisse nach Deutschland ist nicht vorgesehen.

Im Resümee seines Vortrages sagte Dr. Schulz, daß viel Geduld, Beharrlichkeit und natürlich auch Geld in den vergangenen Jahren notwendig waren, um das Joint Venture soweit wie geschildert vorzubereiten. Zur Klärung zahlreicher Detailfragen sei es zudem unbedingt notwendig gewesen, daß ein Consultingbüro vor Ort den ständigen Kontakt zu den chinesischen Partnern in Beijing und Shanghai gehalten hat.

\section{C.3. Finanzierung und Erschließung von Auslands- märkten für brandenburgische und Berliner Un- ternehmen unter besonderer Berücksichtigung des chinesischen Marktes \\ Dipl.-Kfm. Kurt Görger, Leiter der Gruppe Ostasien bei der Berliner Bank $A G$}

Mit seinem ausführlichen und detaillierten Vortrag machte Herr Görger deutlich, daß heutzutage Geschäfte mit Partnern in der Volksrepublik China wesentlich einfacher geworden sind - im Vergleich zur Zeit vor 10 oder 20 Jahren. Die meisten deutschen Geschäftsbanken, so auch die Berliner Bank AG, verfügen inzwischen über ein gut ausgebautes Netz von Korrespondenzbanken in der VR China (vgl. Tagungsbeitrag, S. 5). Insgesamt wird von internationalen Agenturen die Kreditwürdigkeit der chinesischen Volkswirtschaft als „gut“ eingestuft - die Währungsreserven der VR China im Jahre 1996 betrugen 107 Mrd. US Dollar. Im Vergleich dazu betrugen die Währungsreserven Hongkongs 64 Mrd. US Dollar und jene Taiwans 88 Mrd US Dollar (vgl. Tagungsbeitrag, S. 1).

Nicht alle Banken in der VR China sind befugt, Geschäfte in ausländischer Währung zu tätigen. Deshalb sei zu empfehlen, bei der Zusammenarbeit mit chinesischen Partnern von Anfang an auch die Wahl eines autorisierten chinesischen Finanzinstitutes zu klären. Dokumentenakkreditive können nur in China fällig gestellt werden, so daß eine chinesische Bank die Haftung übernehmen muß. Von Seiten des deutschen Verkäufers ist unbedingt darauf zu achten, daß das Akkreditiv mit den dazugehörigen Dokumenten korrekt ausgefüllt wird.
Die Dokumente werden von chinesischer Seite penibelst geprüft, jeder Formfehler gefährdet die Gültigkeit des gesamten Akkreditives.

Exportgeschäfte, über Akkreditive abgesichert, bezeichnete Herr Görger als Test für den Einstieg in den chinesischen Markt. Sofern sich die Geschäftsbeziehung positiv entwickelt, so daß fristgerechte Zahlungseingänge der chinesischen Kunden erkennbar sind, ist im weiteren auch das Factoring/Forfaiting durch die deutsche Geschäftsbank möglich. Kapitalintensivere Strategien (vgl. WONNEBERGER/SWAT et al. 1998) bis hin zur Etablierung eines hundertprozentigen Tochterunternehmens in der VR China sind heutzutage selbst ohne Hermesbürgschaft finanzierbar - eine entsprechende Bonität des deutschen und/oder chinesischen Unternehmens vorausgesetzt.

Summa summarum machte Herr Görger in seinem Vortrag deutlich, daß eine weitere positive Entwicklung der Geschäftsbeziehungen zwischen deutschen und chinesischen Partnern - Banken wie Unternehmen - möglich sei, welche durch den geplanten Beitritt der VR China zur World Trade Organisation WTO neue Impulse erhalten wird.

\section{C.4. Angebote der Bundesstelle für Außenhandels- information \\ Dr. Inge Toschev, Bereichsleiterin für Auslandsmärkte bei der Bundesstelle für Außenhandelsinformation (BfAl)}

Frau Dr: Toschev stellte in ihrem Beitrag die Bundesstelle für Außenhandelsinformation als ein kundenorientiertes, öffentliches Unternehmen vor, welches jährlich über 300.000 Anfragen zur Außenwirtschaft aus Wirtschaft und Gesellschaft beantwortet. Als Basis der Außenhandelsinformation dienen nicht nur ausländische Quellen (Zeitschriften u.a. Publikationen), sondern auch ein Netz von 48 Korrespondenten in den wichtigsten Märkten weltweit. Von daher ist es möglich, daß die BfAl auch Anfragen ihrer Kunden aufnimmt und aktuelle Recherchen vor Ort von ihren Korrespondenten durchführen läßt.

Für ihre Kunden gibt die BfAl regelmäßig eine umfangreiche Reihe von Publikationen heraus, deren Inhalte auch über eine CD-ROM-Datenbank sowie eine OnlineDatenbank verfügbar sind. Frau Dr. Toschev beschrieb das Informationsverhalten der BfAI-Kunden als fall- oder aktualitätsorientiert. Fallorientiert soll heißen, daß die Unternehmen Geschäftskontakte oder Kundenanfragen aus dem Ausland bekommen haben, zu denen sie nunmehr Hintergrundinformationen wünschen. Aktualitätsorientiert soll heißen, daß die Berichterstattung in den Medien - beispielsweise über eine Reise des Bundeskanzlers in die VR China oder nach Argentinien - den Wunsch nach weiteren Länderinformationen auslöst. Eine kontinuierliche Nutzung der BfAl-Informationen durch ein Abonnement von Zeitschriften u. a. Publikationen, CD-ROM, Datenbank ist derzeit, zumindest bei KMU, selten.

Von daher formulierte Frau Dr. Toschev als Ziele für die Arbeit der BfAl in den nächsten Jahren,zum einen die Erhöhung des Bekanntheitsgrades in den neuen Bundesländern, zum anderen bundesweit die Erhöhung der Zahl ihrer Abonnenten. 


\section{C.5. Öffentliche Beratung und Förderung bei der Erschließung von Auslandsmärkten \\ Dipl.-Jap. Frank Beyer, Asien-Referent bei der Wirt- schaftsförderung Brandenburg $\mathrm{GmbH}$}

Den abschließenden Beitrag bei unserem Workshop hielt Herr Dipl.-Jap. Beyer von der Wirtschaftsförderung Brandenburg $\mathrm{GmbH}$, der zunächst einen Überblick über das Außenwirtschaftskonzept des Landes Brandenburg gab. Die Schwerpunkte für die nächsten Jahren liegen zunächst einmal auf der Förderung der Messepräsentation. Als weitere Veranstaltungen zur Außenwirtschaft wurden und werden Kontaktbörsen, Unternehmerreisen und Bietergemeinschaften veranstaltet. Nach Meinung von Herrn Beyer sollte die Definition der Prioritäten nach Wirtschaftszweigen und Ländern für das Außenwirtschaftskonzept in möglichst enger Abstimmung mit den brandenburgischen Unternehmen und ihren Fachverbänden erfolgen. Eine derart koordinierte Prioritätensetzung gelingt, nach den Aussagen von Herrn Beyer, bei der Vielzahl der Institutionen (Bundesregierung und EU-Kommission eingeschlossen) und Verbände in Deutschland manchmal nur mühsam.

Herr Beyer kontrastierte diese Aussagen mit seinen langjährigen Erfahrungen aus der Zusammenarbeit mit der JETRO, der wichtigsten japanischen Organisation für Außenhandelsinformation und Außenwirtschaftsförderung. In deren Tokioer Zentrale sind, nach seinen Erfahrungen, auch um 20 Uhr noch kompetente Gesprächspartner für ausländische Investoren oder japanische Unternehmer anwesend.

Ferner berichtete Herr Beyer über die Präsentation von 15 Brandenburger KMU während einer Februarwoche 1997 in einem Tokioer Kaufhaus. Einer der Teilnehmer dieser Japanpräsentation, der Ofenbaumeister Herr Stich, berichtete bei unserem Workshop von interessanten und erfolgversprechenden Geschäftskontakten. Abschließend ging Herr Beyer noch auf die seit Jahren geäußerte Kritik an den japanischen Handelspraktiken ein. Als Reaktion hierauf wird die JETRO seit Beginn der 90er Jahre auch verstärkt aktiv in der Unterstützung von Unternehmen beim Markteinstieg in Japan, wozu verschiedene Förderprogramme konzipiert und realisiert wurden. Um nur ein Beispiel zu nennen: In einem Business-Support-Center wird den Unternehmen ein Büro für zwei Monate kostenlos zur Verfügung gestellt. Diese und andere Unterstützungen sind für brandenburgische und Berliner Unternehmen rasch und einfach über das Berliner Büro der JETRO nutzbar - ein Hinweis, der von den Teilnehmern unseres Workshops mit großem Interesse aufgenommen wurde.

\section{Zusammenfassung}

In den Beiträgen von Frau Thomsen und Herrn Schulz wird unseres Erachtens nach deutlich, daß diesen Unternehmen die Erschließung internationaler Märkte (außerhalb Europas) ohne eine effiziente und kompetente Außenwirtschaftsförderung wesentlich schwerer gefallen wäre. Die notwendige Effizienz und Kompetenz in der Außenwirtschaftsförderung sind nicht allein abhän- gig von der Höhe der eingesetzten Fördergelder. Bei unserem Workshop hatten die Diskussionsbeiträge der 23 teilnehmenden Unternehmen nur selten Fragen der Kontaktfindung oder der Finanzierung im internationalen Geschäft zum Gegenstand. Die meisten Beiträge der drehten sich um die Frage, wie kompetentes Personal in den Unternehmen für die Erschließung internationaler Märkte ausgebildet und effizient eingesetzt werden könnte - letztlich eine Kernfrage für die internationale Wettbewerbsfähigkeit von KMU (vgl. oben, Abschnitte A.3.ff), welche uns bei den Interviews im Rahmen des Forschungsprojektes aufgefallen war. Gerade der Beitrag von Frau Thomsen macht deutlich, welcher Arbeitsaufwand in der Vorbereitung einer Auslandsreise bzw. Messepräsentation steckt. Idealerweise sollte sich ein Mitarbeiter für den Zeitraum von 6 bis 12 Monaten auf derartige Aufgaben konzentrieren können. Hierfür jedoch ist in den wenigsten KMU ein ausreichendes finanzielles Budget vorhanden.

Nach unserer Auffassung wäre zur Lösung des personellen Engpasses für internationale Aufgaben in KMU zu diskutieren, wieweit staatlich finanzierte, externe Berater einem oder mehreren Unternehmen für eine definierte Anzahl von Monaten zur Verfügung gestellt werden. Diese Berater vor Ort, mit guten Kenntnissen der internationalen Leistungsfähigkeit der brandenburgischen Unternehmen und der internationalen Märkte, könnten verstärkt bei den Technologietransferstellen der Fachhochschulen und Universitäten, den Kammern und Fachverbänden der gewerblichen Wirtschaft beschäftigt werden, um die Erschließung internationaler Märkte für die brandenburgischen Unternehmen der verschiedenen Wirtschaftszweige zu betreiben. Die Unternehmen sollten sich dabei auf die Kernaufgaben der Unternehmensführung (Qualitätsmanagement, Personalführung, Marketing) konzentrieren können, während die Berater im wesentlichen die haushaltsrechtlich notwendigen „bürokratischen Riten" der Projektanträge, -berichte und -abrechnungen für jedwede Fördermaßnahme bei der Erschließung internationaler Märkte zu leisten hätten. Dies erscheint uns besonders wichtig, um die in den Interviews deutlich spürbare Distanz der Unternehmen zu jeder Art von Politik inkl. Wirtschaftsförderung zu verringern (vgl. HEMMERSBACH, RIEDEL).

\section{Literaturhinweise}

Backhaus, Klaus (1992): Investitionsgütermarketing; 3. Auflage, München

Bergemann, Niels und Sourisseaux, Andreas L.J. (Hrsg.) (1992): Interkulturelles Management; Heidelberg BMWi Bundesministerium für Wirtschaft (1996): Investitionschancen und Erfahrungen kleiner und mittlerer Unternehmen im asiatisch-pazifischen Raum; Bonn Hemmersbach, Andreas (1993): Wirkungen von Instrumenten der Technologie- und Exportförderungspolitik für kleine und mittlere Unternehmen; Frankfurt/Main u.a. Henning, Christine und Schiewe, Dirk (1997): Chancen und Barrieren von kleinen und mittleren Unternehmen aus Berlin und Brandenburg beim Eintritt auf den asiatischen Markt; Diplomarbeit an der FHTW Berlin 
Holzmüller, Hartmut H. (1995): Konzeptionelle und methodische Probleme in der interkulturellen Management- und Marketingforschung; Stuttgart

Hofstede, Geert (1992): Die Bedeutung von Kultur und ihren Dimensionen im Internationalen Management; in: Kumar, Brij Nino und Haussmann, Helmut: Handbuch der internationalen Unternehmenstätigkeit; München 1992

Hofstede, Geert (1993): Interkulturelle Zusammenarbeit - Kulturen, Organisationen, Management; Wiesbaden Kulicke, Marianne (1993): Chancen und Risiken junger Technologieunternehmen; Heidelberg

Köglmayr, Hans-Georg (1990): Die Auslandsorientierung von Managern als strategischer Erfolgsfaktor; Berlin Mïller, Stefan (1991): Die Psyche des Managers als Determinante des Exporterfolges; Stuttgart

Mugler, Josef (1995): Betriebswirtschaftslehre der Kleinund Mittelbetriebe; 2. Auflage, Wien und NewYork

Obbelode, Frank (1993): Strategisches Marktauswahlverhalten mittelständischer Unternehmen auf internationalen Märkten; Frankfurt/Main u. a.

Riedel, Harald (1993): Investitionsförderung mittelständischer Unternehmen in strukturschwachen Regionen; Frankfurt/Main u. a.

Simon, Hermann (1996): Die heimlichen Gewinner (Hidden Champions); Frankfurt/Main u. a.

Statistisches Bundesamt (1997): Zusammenfassende Übersichten für den Außenhandel - Dezember 1996; Fachserie 7, Reihe 1; Wiesbaden

Usunier, Jean Claude und Walliser, Björn (1993): Interkulturelles Marketing; Wiesbaden

Wonneberger, Bernd/Könecke, Hans/Weickert, Sven (1997): Asien - Wachstumsmärkte für Brandenburger Unternehmen? in: Wissenschaftliche Beiträge der TFH Wildau, Heft 1/1997

Wonneberger, Bernd/Könecke, Hans/Swat, Rudolf (1998): Die Wahl der Strategie für den Eintritt auf asiatische Märkte durch Brandenburger und Berliner Unternehmen; in: Wissenschaftliche Beiträge der TFH Wildau, Heft $1 / 1998$

\section{Verfasser}

Prof. Dr. Bernd Wonneberger

Dipl.-Psych. Hans Könecke

Technische Fachhochschule Wildau

Institut für internationales Marketing und Unter-

nehmensführung

Bahnhofstraße

15745 Wildau

Tel. +49 (0) 3375 - 508325

Fax +49 (0) $3375-508388$ 